\title{
Evidence of a Time Constant Associated With Movement Patterns in Six Mammalian Species
}

\author{
Geoffrey E. Gerstner \\ University of Michigan, Ann Arbor \\ Louis J. Goldberg \\ State University of New York, Buffalo
}

\begin{abstract}
Human psychophysical studies have provided evidence of a short duration time constant associated with perceptual tasks. This time constant is approximately $3 \mathrm{~s}$ in duration, and evidence suggests that it represents a central neural mechanism that functions to integrate "successive events into a Gestalt" in order to create a "subjective present." Recent studies have found a $3 \mathrm{~s}$ time constant in human and chimpanzee movement patterns, suggesting that a similar mechanism subserves both human perceptual and primate motor skills. These studies have focused exclusively on humans and chimpanzees; therefore, it is unclear whether this time constant represents a characteristic derived in the primate order or an ancestral characteristic found in many different mammalian orders. The current study looked for evidence of a $3 \mathrm{~s}$ time constant associated with movement patterns in six mammalian species representing three non-primate orders. The results showed that all six species' movement pattern event durations averaged about $3 \mathrm{~s}$, and that there were no significant differences in the mean event durations among the species. Thus, the $3 \mathrm{~s}$ time constant originally found in human perceptual and primate motor skills is common among many mammalian orders and probably represents the operation of an ancestral neural mechanism.
\end{abstract}

KEY WORDS: Movement patterns; Event durations; Mammal; Artiodactyla; Carnivora; Marsupialia; Giraffe; Okapi; Roe deer, Eastern gray kangaroo; Red panda; Raccoon.

\section{INTRODUCTION}

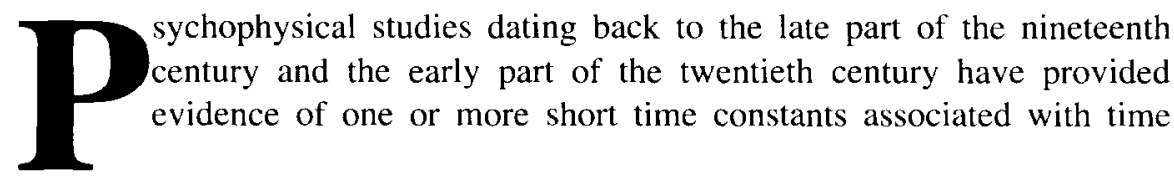

Received March 31, 1993; accepted March 3, 1994.

Address reprint requests to: Geoffrey E. Gerstner, 2351 Neuroethological Systems Science Laboratory, University of Michigan School of Dentistry, Ann Arbor, MI 48109-1078. 
perception in humans (for reviews, see Pöppel 1978; Woodrow 1951). One of these time constants, which was found to be on the order of a few seconds, appeared to be associated with a "unitary awareness of duration" (Woodrow 1951), called the "psychological present" (Woodrow 1951) or "subjective present" (Pöppel 1978).

More recent work (Pöppel 1973) in the area of human time perception has continued to show that "there is something peculiar about the subjective experience of temporal intervals in the range of $2 \mathrm{~s}$ " (Pöppel 1978). Evidence suggests that humans experience temporal intervals in the $2-5 \mathrm{~s}$ range as units. whereas longer temporal units can be estimated symbolically only and cannot be directly perceived (Pöppel 1978). Although the physiological mechanism associated with the 2-5 s subjective experience of time is not known, evidence suggests that an oscillatory, endogenous central neural mechanism is involved (Pöppel 1972, 1978).

Interestingly, this time constant does not appear to be limited to time perception. Pöppel's review (1978) indicates that many investigators have also found temporal patterns in speech that seem to have durations on the order of 2-3 s (Turner and Pöppel 1983). Based on this evidence, Pöppel (1978) suggested that the temporal patterns in speech may serve as the basis upon which the subjective present is built. Whether the subjective present and human rhythmic speech patterns are subserved by similar neural mechanisms, or whether the subjective present emerges as a consequence of the periodic nature of speech is unclear (Pöppel 1978, 1988). What is clear, and what has been emphasized by Pöppel (1978) is that both speech and perceptual skills appear to share a similar time constant.

Based largely on Pöppel's (1978) suggestions regarding the 2-5 s time constant that is shared by both the subjective present and rhythmic patterns in speech, Schleidt (1988) and Schleidt et al. (1987) posited that this time constant might also be found in "overt behavior" of humans. Using a variety of carefully selected movement paradigms observed in humans representing four distinct cultures, these investigators found evidence of a $3 \mathrm{~s}$ time constant governing the duration of an episode of repetitive movements (Schleidt 1988; Schleidt et al. 1987). This work has since been extended to include human nonrepetitive movements, where it has been shown that $93 \%$ of such movements have a duration of 2-3 s (Schleidt and Feldhütter 1989; Feldhütter et al. 1990). Schleidt (1988) concluded that "these findings indicate the influence of a time-regulating mechanism contained in the central nervous system that operates not only in the domains of perception and acoustic communication ... but also in movement patterns of everyday behavior."

Recently, Kien et al. (1991) have shown that hand movements in chimpanzees also are on the order of a few seconds in duration. These results suggest that human perceptual, language, and motor abilities evolved from elaborations of features that already existed in the pongid brain (Kien et al. 1991). Although there is an interesting correlation between the time durations found in human perceptual and motor skills, and in chimpanzee motor skills it 
has yet to be proved whether such skills represent the operation of similar physiological mechanisms. Nevertheless, the fact that human perceptual, as well as human and chimpanzee motor skills have a similar time constant is compelling, and further investigation is in order to determine whether these phenomena are subserved by similar physiological mechanisms.

These previous experiments have focused exclusively on humans and chimpanzees. As a result, it is unclear whether the $3 \mathrm{~s}$ temporal segmentation in perception and motor skills (Pöppel 1988; Schleidt 1988; Schleidt et al. 1987; Kien et al. 1991) are derived features among pongids and hominids or whether this temporal segmentation is sharcd among a broader range of mammalian species.

The probable reason as to why work in non-primate species has not been done to date is as follows. Most investigations up until very recently have focused on the time constant's role in human time perception. Obviously, such perceptual skills would be difficult to investigate in non-human species. Only in the past few years has this work been extended to include overt human motor skills (Schleidt 1988; Schleidt et al. 1987). Clearly, only after the time constant was found in overt human motor skills did it become reasonable to look for a similar time constant in animal movement patterns, and it would seem logical that such non-human studies would begin with species, like chimpanzees, that are closely related to humans (Kien et al. 1991).

To our knowledgc, movement pattern studies similar to the ones discussed previously have not been done to date using non-primate mammals. Therefore, the current study was undertaken in order to determine whether non-primate mammalian species showed evidence of a $3 \mathrm{~s}$ time constant in their overt movement patterns.

\section{METHODS}

\section{Animals}

Twenty-seven animals representing three mammalian orders, six different mammalian species, and eight subspecies were studied (Table 1). The three mammalian orders represented were Artiodactyla, Marsupialia, and Carnivora. Representing the order Artiodactyla were Roe deer (Capreolus capreolus, $n=4$ ), okapis (Okapi johnstoni, $n=4$ ), and 2 giraffe subspecies, viz., Masai giraffes (Giraffa camelopardalis tippelskirchi, $\mathrm{n}=3$ ) and Rothschild's giraffes (G. c. rothschildi, $n=3$ ). Representing the order Marsupialia were Eastern gray kangaroos (Macropus giganteus giganteus, $n=6$ ). Finally, the order Carnivora was represented by Common raccoons (Procyon lotor lotor, $n=3$ ), and two subspecies of Lesser or Red pandas, viz., Styan's pandas (Ailurus fulgens styani, $n=2)$ and A. f. fulgens $(n=2)$. These six species represented a broad spectrum of mammalian characteristics and evolutionary histories (Eisenberg 1981) and therefore provided general inference regarding movement pattern differences that exist among mammals. 
Table 1. Animals Listed by Subspecies, Gender, Age, Housing, and Feeding Schedule

\begin{tabular}{|c|c|c|c|c|c|}
\hline Animals & Gender & $\begin{array}{l}\text { Age } \\
\text { (yrs) }\end{array}$ & Housinga & $\begin{array}{l}\text { Enclosure } \\
\text { sire }\end{array}$ & Ferding schedule: \\
\hline \multirow[t]{4}{*}{ Roc deer } & 个 & 5 & SDZ & $170 \mathrm{~m}^{2}$ & \multirow{4}{*}{$\begin{array}{l}\text { 0530-1000 oats, alfalfa pellets, vegetables. } \\
\text { hay }\end{array}$} \\
\hline & f & 5 & SDZ & $170 \mathrm{~m}^{2}$ & \\
\hline & $\mathrm{m}$ & 5 & SD7. & $170 \mathrm{~m} ?$ & \\
\hline & $\mathrm{m}$ & 4 & SDZ & $130 \mathrm{~m}^{2}$ & \\
\hline \multirow[t]{4}{*}{ Okapi } & f & 2 & WAP & $3400 \mathrm{~m}^{2}$ & \multirow{4}{*}{$\begin{array}{l}0700-0900 \text { allalla pellets, hay } \\
1000-1130 \text { acacia browse } \\
1600-1800 \text { acacia browse, alfalfa pellets, hay }\end{array}$} \\
\hline & $\mathrm{m}$ & 15 & WAP & $3400 \mathrm{~m}^{2}$ & \\
\hline & $\mathrm{m}$ & 3 & WAP & $1100 \mathrm{~m}^{2}$ & \\
\hline & f & 12 & WAP & $1100 \mathrm{~m}^{2}$ & \\
\hline \multirow[t]{3}{*}{ Masai giraffe } & f & 3 & SDZ & $350 \mathrm{~m}^{2}$ & \multirow{3}{*}{$\begin{array}{l}0700-(09,30 \text { acacia browse } \\
1600-1800 \text { vegetables }\end{array}$} \\
\hline & f & 4 & SDZ & $350 \mathrm{~m}^{2}$ & \\
\hline & $\mathrm{m}$ & 13 & SDZ & $350 \mathrm{~m}^{2}$ & \\
\hline \multirow{3}{*}{$\begin{array}{l}\text { Rothschild's } \\
\text { giratfe }\end{array}$} & 1 & & WAP & 128 acres & \multirow[t]{3}{*}{$0800-1100$ acacia browse, alfalfa pellets, hay } \\
\hline & 1 & $5-12^{b}$ & WAP & 128 acres & \\
\hline & $\mathrm{m}$ & & WAP & 128 acres & \\
\hline \multirow[t]{6}{*}{ Kangaroo } & 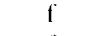 & 8 & SDZ. & $170 \mathrm{~m}^{2}$ & \multirow[t]{6}{*}{$(0900-1130$ vegetables, alfalfa pellets, hay } \\
\hline & $r$ & 11 & SDZ & $170 \mathrm{mI}^{2}$ & \\
\hline & $\mathrm{m}$ & 11 & SDZ & $170 \mathrm{~m}^{2}$ & \\
\hline & $\mathrm{m}$ & 0.3 & SDZ & $170 \mathrm{~m}^{2}$ & \\
\hline & $\mathrm{m}$ & 12 & L.AZ & $170 \mathrm{~m}^{2}$ & \\
\hline & i & 3 & $1 . A Z$ & $170 \mathrm{~m}^{2}$ & \\
\hline \multirow[t]{3}{*}{ Raccoon } & $m$ & 2 & PZ & $220 \mathrm{~m}^{2}$ & \multirow[t]{3}{*}{ 0800-0830 eggs, fish. fruit, hard dog food } \\
\hline & $\mathrm{m}$ & 2 & $\mathrm{PZ}$ & $220 \mathrm{~m}^{2}$ & \\
\hline & $\mathrm{m}$ & 2 & $\mathrm{P} Z$ & $220 \mathrm{~m}^{2}$ & \\
\hline \multirow[t]{2}{*}{ Styan's panda } & $i$ & 9 & SD7. & $20 \mathrm{~m}^{2}$ & \multirow{4}{*}{$\begin{array}{l}0930-1030 \text { bamboo } \\
1230-1300 \text { fruit, gruel } \\
\text { (1800-1100 hamboo, gruel, monkey hiscuits. } \\
\text { fruit }\end{array}$} \\
\hline & i & 10 & SDZ & $20 \mathrm{~m}^{2}$ & \\
\hline \multirow[t]{2}{*}{ Fulgens panda } & $i$ & 8 & L..A\% & $23 \mathrm{~m}^{2}$ & \\
\hline & $\mathrm{m}$ & 8 & LAZ & $23 \mathrm{~m}^{2}$ & \\
\hline
\end{tabular}

"Abhreviations: SDZ. San Diego Zoo: WAP, San Diego Wild Animat Park; LAZ, Greater Los Angeles Zoo; PZ. Phoenix Zoo).

"Ages of the Rothschild's girates were nol known detinitively.

Table 1 shows the genders and ages of the animals in the study as well as where each animal was housed, the size of the enclosure in which the animal was housed, food supplies, and the time of day the food supplies were replenished. Animals were kept at either the San Diego Zoo, the San Diego Wild Animal Park, the Greater Los Angeles Zoo, or the Phoenix Zoo (Table 1). In most cases, all individuals of a given species occurring in the same exhibit were included in the study. However, in the case of the okapis, Rothschild's giraffes, and the Greater Los Angeles Zoo kangaroos (Table 1) individuals were randomly selected from larger species groups for inclusion in the study by using randomization software developed in the laboratory.

All animals had ad libitum access to water and food during daylight hours. Although animals often depleted some food supplies before day's end, other food sources including naturally occurring food sources were always available to the animals. Naturally occurring items included leaves, grass, flowers, and bark for the giraffes, okapis, deer, and kangaroos; grass and leaves for the pandas; and roots and invertebrates for the raccoons.

Animals were kept in relatively rich environments, which included a variety of flora, water pools, and varying terrain. The deer, okapis, and Rothschild's giraffes were housed with other mammalian and avian species as 
well, thus further enriching the environment. All animals except for the okapis occurred in public access enclosures; the okapi exhibits had no direct public access.

During the study, several births occurred. Both female okapis and the 4-year-old Masai giraffe bore calves, and one of the Roe deer bore two healthy fawns. These five offspring were not studied; however, the adult interactions with the offspring were frequently videotaped and studied.

It is important to note that within 6 months of the study's completion, the 11-year-old male and the 8-year-old female kangaroos from the San Diego Zoo died of encephalitis secondary to lumpy jaw (Boever and Leathers 1973); however, the data from these two kangaroos did not appear to be altered by the disease.

\section{Videotaping Methods}

Videotaping sessions. Each of the 27 animals was videotaped on four separate occasions during a 1.5 -year period. A videotaping session began between 0500-0830 hours and usually ended between 1700-2030 hours. In general, the duration of a videotaping session was dictated either by available light levels or zoo policies regarding before- and after-hour observation studies. On four occasions, okapis and giraffes were put in night houses in the early afternoon as a result of high winds or heavy rain, at which time the videotaping session was stopped earlier than 1700 hours.

Each videotaping session lasted for 6-15 h with the only interruption in taping occurring during the $45 \mathrm{~s}$ period required to unload and reload the camcorder with videotape. This interruption occurred once every $6 \mathrm{~h}$, or in other words 1-2 times per videotaping session. An average videotaping session lasted $10.5 \mathrm{~h}$. This length of time helped to maximize the variation in movement patterns observed at different times of the day. Over $1100 \mathrm{~h}$ of videotaping occurred during the study.

Rules governing which animals were videotaped and when. A strict set of rules governed the videotaping procedures; these rules have been described fully elsewhere (Gerstner 1992). These rules were used primarily to maximize movement pattern variability within and between videotaping sessions. Briefly, each of the four separate videotaping sessions for each animal had to occur during a different season so that season-specific movement patterns were more likely to be videotaped. Furthermore, because one animal's behavior can strongly influence the behavior of neighboring animals (Wilson 1980, chap. 2), and since ultradian behavior patterns tend to be similar over a 4-day period (Kavanau and Rischer 1968; Kavanau and Peters 1974), at least 5 days had to pass between videotaping sessions of two different animals housed in the same or adjoining enclosures.

Environmental stimuli can entrain an animal's behavior patterns (Gwinner 1986, chap. 4), and zoo routines, e.g., feeding schedules, pen cleaning sched- 
ules, tour bus schedules, park opening and closing times, etc., may entrain zoo animal behavior patterns. Therefore, in order to maximize movement pattern variation in the animals, the four videotaping sessions for an individual animal had to: (1) occur on different days of the week, (2) occur on both weekends as well as weekdays, and (3) involve a different zoo keeper in charge of a given animal's care at each videotaping session. Zoo routines as well as public attendance parameters, e.g., numbers of zoo visitors, family attendance vs. school group attendance, etc., were maximally varied by these rules; therefore, it was believed that behavior patterns that were entrained by stimuli associated with public and zoo routines would also be maximally varied by employing these rules.

Prior to the study's onset, the order for videotaping at each enclosure was randomized across all four seasons using software developed in the laboratory. The order in which the animals in each enclosure were to be videotaped was also randomized for the first season. In ensuing seasons, the sequence in which the animals in an enclosure were videotaped was altered so that no two animals were videotaped in the same sequence in consecutive seasons. In other words, if animals $\mathrm{A}, \mathrm{B}$, and $\mathrm{C}$ occurred in one enclosure and were taped in the order $\mathrm{A}-\mathrm{B}-\mathrm{C}$ during one season, then in the next season, $\mathrm{C}$ could not follow $\mathrm{B}$, nor could $\mathrm{B}$ follow $\mathrm{A}$. This rule helped minimize any habituation or entrainment effects associated with the investigator operating the camera.

\section{Ethograms, Movement Patterns, and Movement Pattern Categories}

A major methodological objective of this study was to account for every single movement of every single appendage or body part of each animal over 6-15 h time spans. In order to do this, ethograms or lists of movement pattern categories for each species had to be created. Movement patterns were defined as visually observable patterns of motor activity in which a given animal engaged. Each movement pattern category defined a unique movement pattern. A movement pattern event was defined as a single, discrete, continuous occurrence of a given movement pattern. An event was discrete in that it had a visually observable onser and offset (see Figure $1 B$ ).

The ethogram movement pattern categories for each species appear as Table 2. Because similar wording can be used to describe movement patterns made by different species, e.g., locomotion can be used to describe movement patterns made by each of the six species, often the same movement pattern category was used to describe movements made by different species. With this in mind, Table 2 shows the entire list of movement pattern categories for all species combined in the first column, and the remaining six columns indicate in which species movement patterns represented by a given category were observed.

Movement patterns ranged from fairly simple movements, e.g., "adjusting or moving ear(s)," to complex highly variable movements, e.g., "physical 
A

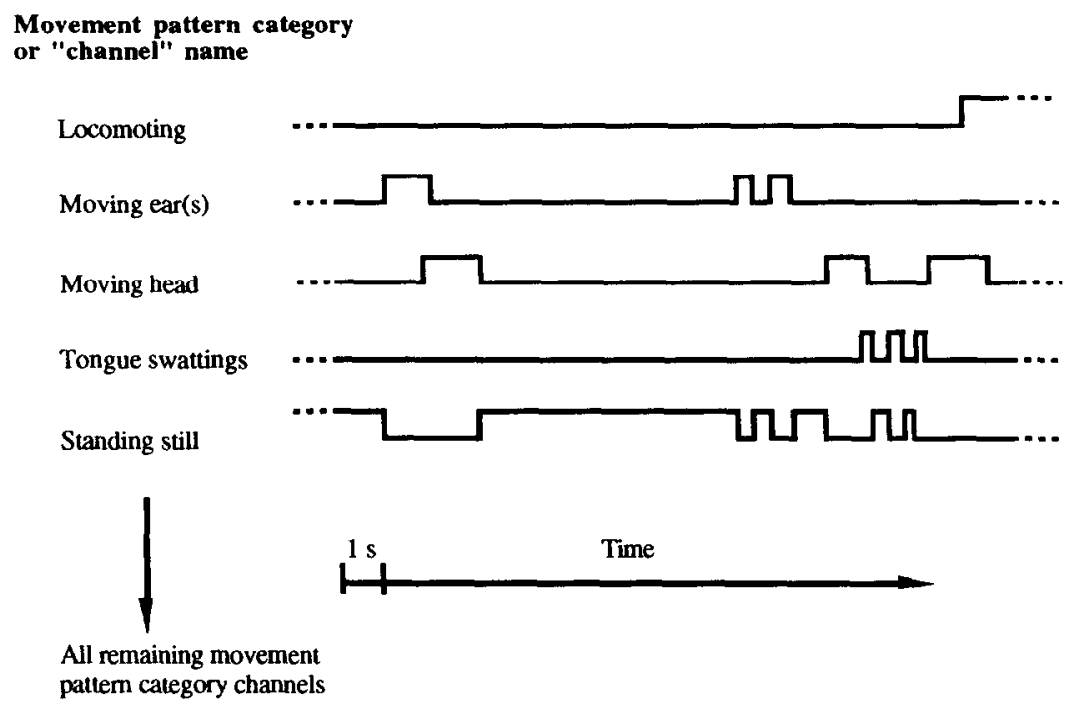

B

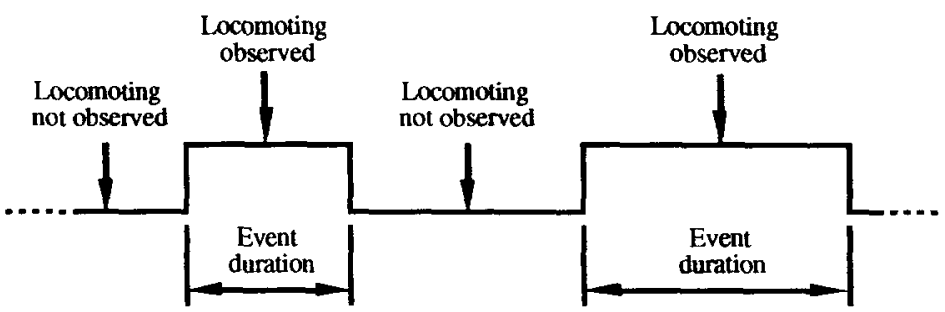

Time

FIGURE 1. Summary of ethogram data parameters. (A) Five hypothetical movement pattern categories or "channels" are shown. Each species engaged in motor activity throughout the day that was describable by $59-80$ such movement pattern categories. (B) Event duration was defined as the time from a specific movement pattern category event onset to the same event's offset.

wrestling (play) with conspecific" (Table 2). Movement patterns also ranged from rhythmic movement patterns as in bouts of locomotion or chewing to nonrhythmic movements as when an animal adjusted its limbs, head or body during a rest period.

The final ethogram movement pattern categories (Table 2) were created so that all movements in which an animal engaged during a 6-15 h videotape session were accounted for by one and only one category. This meant that the ethogram movement pattern categories had to be mutually exclusive and 
Table 2. Ethograms or Movement Pattern Category Names

\begin{tabular}{|c|c|c|c|c|c|c|}
\hline \multirow[b]{2}{*}{$\begin{array}{l}\text { Movement Pattern } \\
\text { Categories }\end{array}$} & \multicolumn{6}{|c|}{$\begin{array}{c}\text { Species in which Movement } \\
\text { Pattern Was Observed }\end{array}$} \\
\hline & $\begin{array}{l}\text { Gir- } \\
\text { affe }\end{array}$ & Okapi & $\begin{array}{l}\text { Roe } \\
\text { Deer }\end{array}$ & $\begin{array}{l}\text { Kan- } \\
\text { garoo }\end{array}$ & $\begin{array}{l}\text { Rac- } \\
\text { coon }\end{array}$ & $\begin{array}{c}\text { Red } \\
\text { Panda }\end{array}$ \\
\hline \multicolumn{7}{|l|}{ Reflex categories } \\
\hline Startle response & $\mathrm{x}$ & $X$ & $x$ & $\mathrm{X}$ & $\mathrm{X}$ & $\mathrm{X}$ \\
\hline \multicolumn{7}{|l|}{${ }^{1}$ Posture categories } \\
\hline Lying still, eyes open & $x$ & $x$ & $x$ & $x$ & $x$ & $x$ \\
\hline Lying still, eyes closed & & & $\mathrm{X}$ & $x$ & $\mathrm{X}$ & $\mathrm{X}$ \\
\hline Lying still, eye status unknown & & & $X$ & $\mathrm{X}$ & $\mathrm{X}$ & $\mathrm{X}$ \\
\hline Lying still, head up & & & & & $X$ & $X$ \\
\hline Lying in pouch, eyes open & & & & $x$ & & \\
\hline Lying in pouch, eyes closed & & & & $\mathrm{X}$ & & \\
\hline $\begin{array}{l}\text { Lying in pouch, eye status } \\
\text { unknown }\end{array}$ & & & & $\mathrm{X}$ & & \\
\hline Standing still, eyes open & $x$ & $X$ & $\mathrm{X}$ & $\mathrm{X}$ & $X$ & $X$ \\
\hline Standing still, eyes closed & & & $x$ & $\mathrm{X}$ & & \\
\hline $\begin{array}{l}\text { Standing still. eye status } \\
\text { unknown }\end{array}$ & $\mathrm{X}$ & $X$ & $x$ & $\mathrm{X}$ & $X$ & $x$ \\
\hline Sitting still, eyes open & & & & & $X$ & $x$ \\
\hline Sitting still, eyes closed & & & & & $X$ & $X$ \\
\hline Sitting still, eye status unknown & & & & & $x$ & $\mathrm{x}$ \\
\hline Bipedal stance & & & & & $\mathrm{X}$ & \\
\hline \multicolumn{7}{|l|}{ Increased sensory vigilance category } \\
\hline $\begin{array}{l}\text { Alert posturing: ears forward } \\
\text { eye gaze forward, } \\
\text { eyelids wide open }\end{array}$ & $x$ & $x$ & $x$ & $\mathrm{x}$ & $x$ & $x$ \\
\hline \multicolumn{7}{|l|}{ Posture adjustment catcgorics } \\
\hline Lying to standing & $\mathrm{X}$ & $x$ & $\mathrm{X}$ & $\mathrm{X}$ & $x$ & $x$ \\
\hline Standing to lying & $\mathrm{x}$ & $x$ & $x$ & $\mathrm{X}$ & $\mathrm{X}$ & $\mathrm{x}$ \\
\hline Lying to sitting & & & & & $x$ & $\mathrm{x}$ \\
\hline Sitting to lying & & & & & $X$ & $\mathrm{X}$ \\
\hline Sitting to standing & & & & & $X$ & $\mathrm{x}$ \\
\hline Standing to sitting & & & & & $x$ & $\mathrm{X}$ \\
\hline Standing to bipedal stance & & & & & $\mathrm{x}$ & \\
\hline Bipedal stance to standing & & & & & $x$ & \\
\hline Adjusting or moving ear(s) & $\mathrm{X}$ & $X$ & $X$ & $X$ & $x$ & $\mathrm{X}$ \\
\hline $\begin{array}{l}\text { Adjusting or moving head } \\
\text { and/or neck }\end{array}$ & $\mathrm{x}$ & $x$ & $\mathrm{X}$ & $x$ & $\mathrm{X}$ & $x$ \\
\hline Adjusting or moving body & $x$ & $\mathrm{X}$ & $\mathrm{x}$ & $x$ & $\mathrm{x}$ & $\mathrm{X}$ \\
\hline Adjusting or moving $\operatorname{limb}(\mathrm{s})$ & & & $\mathrm{X}$ & $\mathrm{X}$ & $x$ & $\mathrm{X}$ \\
\hline $\begin{array}{l}\text { Single step, limb movements } \\
\text { or standing adjustments } \\
\text { involving limb movements }\end{array}$ & $x$ & $x$ & $x$ & $x$ & $\mathrm{x}$ & $x$ \\
\hline Spreading limbs while standing & $\mathrm{X}$ & $X$ & & & & \\
\hline $\begin{array}{l}\text { Changing directions }>90^{\circ} \\
\text { while locomoting }\end{array}$ & $\mathrm{X}$ & $\mathrm{X}$ & $\mathrm{X}$ & $\mathrm{x}$ & $\mathrm{X}$ & $\mathrm{X}$ \\
\hline $\begin{array}{l}\text { Splinting limb movements } \\
\text { (associated with guarding } \\
\text { sore limb) }\end{array}$ & $x$ & & & & & \\
\hline Adjusting or moving tail & $\mathrm{X}$ & $\mathrm{X}$ & & & $\mathrm{x}$ & $\mathrm{X}$ \\
\hline
\end{tabular}


Table 2. (Continued from previous page)

\begin{tabular}{|c|c|c|c|c|c|c|}
\hline \multirow[b]{2}{*}{$\begin{array}{l}\text { Movement Pattern } \\
\text { Categories }\end{array}$} & \multicolumn{6}{|c|}{$\begin{array}{l}\text { Species in which Movement } \\
\text { Pattern Was Observed }\end{array}$} \\
\hline & $\begin{array}{l}\text { Gir- } \\
\text { affe }\end{array}$ & Okapi & $\begin{array}{l}\text { Roe } \\
\text { Deer }\end{array}$ & $\begin{array}{l}\text { Kan- } \\
\text { garoo }\end{array}$ & $\begin{array}{l}\text { Rac- } \\
\text { coon }\end{array}$ & $\begin{array}{l}\text { Red } \\
\text { Panda }\end{array}$ \\
\hline \multicolumn{7}{|l|}{ Visceral maintenance categories } \\
\hline Vacuous chewing & $\mathrm{X}$ & $X$ & $\mathrm{X}$ & $\mathrm{X}$ & $\mathrm{X}$ & $\mathrm{X}$ \\
\hline Licking lips with tongue & $\mathrm{X}$ & $\mathrm{X}$ & $\mathrm{X}$ & $\mathrm{X}$ & $\mathrm{X}$ & $\mathrm{X}$ \\
\hline Tongue protrusion stereotypy & $\mathrm{x}$ & & & & & \\
\hline Sneezing & $\mathrm{X}$ & $\mathrm{X}$ & $\mathrm{X}$ & $\mathrm{X}$ & $\mathrm{X}$ & $\mathrm{X}$ \\
\hline Yawning & & & $\mathrm{X}$ & $\mathrm{X}$ & $X$ & $\mathrm{X}$ \\
\hline Stretching & & & $\mathrm{X}$ & $\mathrm{X}$ & $\mathrm{X}$ & $\mathrm{X}$ \\
\hline Hiccuping/body twitching & $\mathrm{X}$ & $X$ & $\mathrm{X}$ & $\mathrm{X}$ & $\mathrm{X}$ & $\mathrm{X}$ \\
\hline Twitching vibrissae or face & & & & & $\mathrm{X}$ & $\mathrm{X}$ \\
\hline Urinating & $\mathrm{X}$ & $\mathrm{X}$ & $\mathrm{X}$ & $\mathrm{X}$ & $\mathrm{X}$ & $\mathrm{X}$ \\
\hline Defecating & $\mathrm{x}$ & $\mathrm{x}$ & $\mathrm{X}$ & $\mathrm{x}$ & $\mathrm{x}$ & $\mathrm{x}$ \\
\hline \multicolumn{7}{|l|}{$\begin{array}{l}\text { Integumental maintenance } \\
\text { categories }\end{array}$} \\
\hline $\begin{array}{l}\text { Pest defensive tongue } \\
\text { swattings to body }\end{array}$ & $\mathrm{X}$ & $\mathrm{X}$ & $\mathrm{X}$ & & & \\
\hline $\begin{array}{l}\text { Pest defensive tongue } \\
\text { swattings to face }\end{array}$ & $\mathrm{X}$ & $\mathrm{X}$ & & & & \\
\hline $\begin{array}{l}\text { Pesı defensive limb flickings } \\
\text { or stompings on ground }\end{array}$ & $\mathrm{x}$ & $\mathrm{x}$ & $\mathrm{x}$ & $\mathrm{x}$ & & \\
\hline Autosniffing/nose to body & $\mathrm{X}$ & $\mathrm{X}$ & $\mathrm{X}$ & $\mathrm{X}$ & $\mathrm{X}$ & $\mathrm{X}$ \\
\hline Grooming-autolicking & $\mathrm{X}$ & $x$ & $\mathrm{x}$ & $\mathrm{X}$ & $\mathrm{X}$ & $x$ \\
\hline Grooming-autobiting & & & & & $\mathrm{X}$ & $\mathrm{x}$ \\
\hline $\begin{array}{l}\text { Grooming-rubbing face } \\
\text { with paws }\end{array}$ & & & & $\mathrm{X}$ & $\mathrm{X}$ & $\mathrm{x}$ \\
\hline Grooming genitalia & & & & $\mathrm{x}$ & $\mathrm{X}$ & \\
\hline $\begin{array}{l}\text { Raccoon hand "washing" } \\
\text { behavior }\end{array}$ & & & & & $\mathrm{X}$ & \\
\hline $\begin{array}{l}\text { Scratching body or face } \\
\text { with limb }\end{array}$ & & & $\mathrm{X}$ & $\mathrm{x}$ & $\mathrm{X}$ & $\mathrm{x}$ \\
\hline $\begin{array}{l}\text { Rubbing head, horns, antlers, } \\
\text { neck, body, or limbs against } \\
\text { inanimate object }\end{array}$ & $\mathrm{X}$ & $\mathrm{X}$ & $\mathrm{X}$ & $\mathrm{x}$ & $\mathrm{X}$ & $\mathrm{X}$ \\
\hline $\begin{array}{l}\text { Shaking-rhythmic rotatory } \\
\text { head or body movements }\end{array}$ & $\mathrm{X}$ & $\mathrm{X}$ & $\mathrm{X}$ & $\mathrm{X}$ & $\mathrm{X}$ & $\mathrm{X}$ \\
\hline \multicolumn{7}{|l|}{ Locomotory categories } \\
\hline $\begin{array}{l}\text { Locomoting-normative (slow } \\
\text { rhythmical) gait }\end{array}$ & $\mathrm{X}$ & $\mathrm{X}$ & $\mathrm{X}$ & $\mathrm{X}$ & $\mathrm{X}$ & $\mathrm{x}$ \\
\hline $\begin{array}{l}\text { Locomoting-trotting or } \\
\text { running speed gait }\end{array}$ & $\mathrm{x}$ & $\mathrm{X}$ & $x$ & & $\mathrm{X}$ & $\mathrm{x}$ \\
\hline $\begin{array}{l}\text { Locomoting-fast hop. } \\
\text { bipedal gait }\end{array}$ & & & & $\mathrm{x}$ & & \\
\hline $\begin{array}{l}\text { Stereotyped pacing-normative } \\
\text { (slow thythmical) gait }\end{array}$ & $\mathrm{x}$ & $X$ & $\mathrm{X}$ & & $\mathrm{X}$ & $\mathrm{x}$ \\
\hline $\begin{array}{l}\text { Stereotyped pacing - trotting } \\
\text { or running speed gait }\end{array}$ & & $\mathrm{X}$ & $\mathrm{x}$ & & $\mathrm{x}$ & $\mathrm{x}$ \\
\hline Climbing an object or cage & & & & & $\mathrm{X}$ & $\mathrm{X}$ \\
\hline $\begin{array}{l}\text { Swimming - rhythmic paddling } \\
\text { movements with forelimbs }\end{array}$ & & & & & $\mathrm{X}$ & \\
\hline $\begin{array}{l}\text { Jumping from one inanimate } \\
\text { object to another }\end{array}$ & & & & & $\mathrm{x}$ & $\mathrm{x}$ \\
\hline
\end{tabular}


Table 2. (Continued from previous page)

\begin{tabular}{|c|c|c|c|c|c|c|}
\hline \multirow[b]{2}{*}{$\begin{array}{l}\text { Movement Pattern } \\
\text { Categories }\end{array}$} & \multicolumn{6}{|c|}{$\begin{array}{l}\text { Species in which Movement } \\
\text { Pattern Was Observed }\end{array}$} \\
\hline & $\begin{array}{l}\text { Gir- } \\
\text { affe }\end{array}$ & Okapi & $\begin{array}{l}\text { Roe } \\
\text { Deer }\end{array}$ & $\begin{array}{l}\text { Kan- } \\
\text { garoo }\end{array}$ & $\begin{array}{l}\text { Rac- } \\
\text { coon }\end{array}$ & $\begin{array}{l}\text { Red } \\
\text { Panda }\end{array}$ \\
\hline \multicolumn{7}{|l|}{ Social-sexual categories } \\
\hline Allosniffing & $\mathrm{X}$ & $\mathrm{x}$ & $\mathrm{X}$ & $\mathrm{X}$ & $\mathrm{X}$ & \\
\hline Allolicking & & $x$ & $\mathrm{X}$ & $\mathrm{X}$ & $\mathrm{X}$ & \\
\hline Nudging conspecific with limbs & $\mathrm{X}$ & $\mathrm{x}$ & $\mathrm{x}$ & $x$ & & \\
\hline Nudging conspecific with body & $\mathrm{X}$ & $\mathrm{X}$ & $\mathrm{X}$ & $\mathrm{X}$ & & \\
\hline $\begin{array}{l}\text { Nudging or butting } \\
\text { conspecific with head, } \\
\text { horns, or antlers }\end{array}$ & $\mathrm{X}$ & $X$ & $\mathrm{X}$ & & & \\
\hline $\begin{array}{l}\text { Flehman (everting lip and } \\
\text { tongue to labial surface } \\
\text { of premaxillary regions) }\end{array}$ & $\mathrm{X}$ & $X$ & $\mathrm{X}$ & $\mathrm{X}$ & & \\
\hline Drinking conspecific urine & $\mathrm{X}$ & $\mathrm{X}$ & $\mathrm{X}$ & $\mathrm{x}$ & & \\
\hline Erection & $\mathrm{X}$ & $\mathrm{X}$ & $\mathrm{X}$ & $\mathrm{X}$ & $\mathrm{X}$ & \\
\hline Mounting conspecific & $\mathrm{X}$ & $\mathrm{X}$ & $\mathrm{X}$ & $\mathrm{x}$ & $\mathrm{X}$ & \\
\hline $\begin{array}{l}\text { Allomasturbating - static } \\
\text { posture }\end{array}$ & & & & & $x$ & \\
\hline $\begin{array}{l}\text { Allomasturbating- } \\
\text { rhythmic arm movements }\end{array}$ & & & & & $x$ & \\
\hline $\begin{array}{l}\text { Mother-infant touching } \\
\text { (this is an "active" behavior. } \\
\text { not simply a result of an } \\
\text { assumed posture) }\end{array}$ & $x$ & $X$ & $\mathrm{X}$ & $\mathrm{X}$ & & \\
\hline $\begin{array}{l}\text { Nursing an infanı or juvenile } \\
\text { (mother behavior, not juvenile } \\
\text { or intant behavior) }\end{array}$ & $\mathrm{X}$ & $\mathrm{X}$ & $x$ & $\mathrm{X}$ & & \\
\hline $\begin{array}{l}\text { Physical "wrestling" } \\
\text { (play) with conspecific }\end{array}$ & & & $x$ & $\mathrm{X}$ & $\mathrm{x}$ & \\
\hline $\begin{array}{l}\text { Play "bucking" (concurrent } \\
\text { body movements, pseudo } \\
\text { rhythmic high amplitude head } \\
\text { and neck movements, and } \\
\text { trotting speed, locomotion) }\end{array}$ & $\mathrm{X}$ & $\mathrm{X}$ & $x$ & $\mathrm{X}$ & $\mathrm{X}$ & \\
\hline $\begin{array}{l}\text { Sparring, fighting, aggressive } \\
\text { movements directed towards } \\
\text { conspecific }\end{array}$ & $\mathrm{X}$ & $\mathrm{X}$ & $\mathrm{X}$ & $x$ & $\mathrm{X}$ & $x$ \\
\hline $\begin{array}{l}\text { Fleeing from aggressive } \\
\text { atlack }\end{array}$ & $\mathrm{X}$ & $x$ & $X$ & $\mathrm{X}$ & $x$ & $\mathrm{X}$ \\
\hline Vocalizing & $x$ & $\mathrm{X}$ & $\mathrm{x}$ & $\mathrm{x}$ & $x$ & $\mathrm{x}$ \\
\hline Nose up display & $x$ & $\mathrm{X}$ & & & & \\
\hline $\begin{array}{l}\text { Neck lowered, rapid head } \\
\text { extension display }\end{array}$ & $\mathrm{x}$ & & & & & \\
\hline $\begin{array}{l}\text { Icad-to-ground } \\
\text { defensive posture }\end{array}$ & & & & & $x$ & \\
\hline $\begin{array}{l}\text { Head-to-ground } \\
\text { submissive posture }\end{array}$ & & & $x$ & & & \\
\hline $\begin{array}{l}{ }^{3} \text { Scent marking, rubbing } \\
\text { urogenital region on } \\
\text { an inanimate object }\end{array}$ & & $\mathrm{x}$ & $\mathrm{x}$ & & $\mathrm{X}$ & $x$ \\
\hline \multicolumn{7}{|l|}{ Ingestive categories } \\
\hline Lapping water & $x$ & $\mathrm{X}$ & $\mathrm{x}$ & $\mathrm{X}$ & $\mathrm{X}$ & $x$ \\
\hline Licking salt lick & $\mathrm{X}$ & $x$ & $\mathrm{X}$ & $\mathrm{X}$ & & \\
\hline $\begin{array}{l}\text { Nursing (juvenile behavior } \\
\text { not mother behavior) }\end{array}$ & $x$ & $\mathrm{X}$ & $x$ & $\mathrm{x}$ & & \\
\hline Sniffing edible items & $\mathrm{X}$ & $x$ & $x$ & $\mathrm{x}$ & $\mathrm{X}$ & $x$ \\
\hline $\begin{array}{l}\text { Licking or grasping edible } \\
\text { items with tongue }\end{array}$ & $\mathrm{x}$ & $\mathrm{x}$ & & & & $\mathrm{x}$ \\
\hline
\end{tabular}


Table 2. (Continued from previous page)

\begin{tabular}{|c|c|c|c|c|c|c|c|}
\hline \multirow[b]{2}{*}{$\begin{array}{l}\text { Movement Pattern } \\
\text { Categories }\end{array}$} & & \multicolumn{6}{|c|}{$\begin{array}{l}\text { Species in which Movement } \\
\text { Pattem Was Observed }\end{array}$} \\
\hline & & $\begin{array}{l}\text { Gir- } \\
\text { affe }\end{array}$ & Okapi & $\begin{array}{l}\text { Roe } \\
\text { Deer }\end{array}$ & $\begin{array}{l}\text { Kan- } \\
\text { garoo }\end{array}$ & $\begin{array}{l}\text { Rac- } \\
\text { coon }\end{array}$ & $\begin{array}{l}\text { Red } \\
\text { Panda }\end{array}$ \\
\hline $\begin{array}{l}\text { Incising, acquiring or carrying } \\
\text { edible items with teeth }\end{array}$ & & $\mathrm{X}$ & $\mathrm{X}$ & $\mathrm{X}$ & $\mathrm{X}$ & $\mathrm{X}$ & $\mathrm{X}$ \\
\hline $\begin{array}{l}\text { Grasping or carrying edible } \\
\text { itemis with limbs }\end{array}$ & & & & & $X$ & $\mathrm{X}$ & $\mathrm{X}$ \\
\hline $\begin{array}{l}\text { Begging for food from zoo } \\
\text { visitors (forelimb movements) }\end{array}$ & & & & & & $X$ & \\
\hline Chewing unknown substance & & $\mathrm{X}$ & $\mathrm{X}$ & $\mathrm{X}$ & $\mathrm{X}$ & $\mathrm{X}$ & $\mathrm{X}$ \\
\hline Chewing monkey biscuits & & & & & & & $\mathrm{X}$ \\
\hline Chewing dog biscuits & & & & & & $\mathrm{X}$ & \\
\hline Chewing alfalfa pellets & & $\mathrm{X}$ & $x$ & & $X$ & & \\
\hline Chewing hay & & $\mathrm{X}$ & $x$ & $\mathrm{X}$ & $\mathrm{X}$ & & \\
\hline Chewing grain & & & & $\mathrm{X}$ & & & \\
\hline $\begin{array}{l}\text { Chewing or lapping gruel } \\
\text { (head is away from source } \\
\text { of gruel) }\end{array}$ & & & & & & $\mathrm{X}$ & $\mathrm{X}$ \\
\hline Chewing grass & & & $\mathrm{X}$ & $X$ & & $x$ & $X$ \\
\hline $\begin{array}{l}\text { Chewing browse (e.g... acacia } \\
\text { or eucalyptus leaves) }\end{array}$ & & $\mathrm{X}$ & $\mathrm{X}$ & $\mathrm{X}$ & $\mathrm{X}$ & & \\
\hline Chewing bamboo leaves & & & & & & & $X$ \\
\hline Chewing tree branches or twigs & & $X$ & $x$ & $x$ & $\mathrm{X}$ & & \\
\hline Chewing tree bark & & $\mathrm{x}$ & $\mathrm{x}$ & $\mathrm{X}$ & $\mathrm{x}$ & & \\
\hline Chewing fruit & & & & & & $\mathrm{X}$ & $\mathrm{X}$ \\
\hline Chewing vegetables & & & & & $\mathrm{X}$ & $X$ & $\mathrm{X}$ \\
\hline Chewing egg & & & & & & $X$ & \\
\hline Chewing fish & & & & & & $\mathrm{X}$ & \\
\hline Ruminating & & $X$ & $\mathrm{X}$ & $\mathrm{X}$ & & & \\
\hline Regurgitating/vomiting & & $X$ & $\mathrm{X}$ & $\mathrm{X}$ & $\mathrm{X}$ & $x$ & \\
\hline \multicolumn{8}{|l|}{$\begin{array}{l}\text { External cnvironment } \\
\text { manipulation categories }\end{array}$} \\
\hline $\begin{array}{l}\text { Object orienting -sniffing, } \\
\text { nosing, licking, or lipping } \\
\text { an inanimate object }\end{array}$ & & $\mathrm{X}$ & $x$ & $x$ & $\mathrm{X}$ & $\mathrm{X}$ & $\mathrm{X}$ \\
\hline $\begin{array}{l}\text { Rhythmic hand movements- } \\
\text { raccoon hand "washing" } \\
\text { behavior }\end{array}$ & & & & & & $X$ & \\
\hline $\begin{array}{l}\text { Rhythmic scratching of } \\
\text { inanimate object with claws }\end{array}$ & & & & & & $\mathrm{X}$ & \\
\hline Pawing ground with hoof & & & & $x$ & $\mathrm{X}$ & & \\
\hline $\begin{array}{l}\text { Rhythmic digging movements } \\
\text { in the ground }\end{array}$ & & & & & & $\mathrm{X}$ & \\
\hline $\begin{array}{l}\text { Pushing, moving or attempting } \\
\text { to move or push inanimate } \\
\text { objects }\end{array}$ & & & & & & $\mathrm{X}$ & \\
\hline${ }^{4}$ Totals: & 113 & 61 & 62 & 66 & 64 & 80 & 59 \\
\hline
\end{tabular}

'Standing = animal's limbs support the animal's weight in a manner typical for the species. For giraffes, okapis, Roe deer, raccoons, and Red pandas, this is a quadrupedal stance; for the kangaroo, this is a bipedal stance that is stabilized by the tail. Lying = animal's weight is supported by the animal's body while the limbs are either in flexion under the animal or are displaced to the animal's side. Sitting = animal's posterior body approximates a lying posture while the forelimbs approximate a standing posture.

${ }^{2}$ Giraffe, okapi, Roe deer, and kangaroos invariably lie still with their heads up, whereas raccoons and Red pandas lie still with their heads up or down. The category "Lying still, head up" was used to distinguish the head attitude of raccoons and pandas. Since "lying still" implied that a giraffe's, okapi"s. deer's, or kangaroo"s head was up. the category "Lying still, head up" was overly descriptive, and was not used for these four species.

${ }^{3}$ This category inciudes the okapis' category of urinating while locomoting through small shrubs.

${ }^{4}$ Totals do not include categories with "eye status unknown" under "Posture categories," or "Chewing unknown substance" under "Ingestive categories." Totals include all movernent pattern categories observed, not just the categories representing movement patterns that were sampled (cf. Figure 2), analyzed, and included in the results (see Methods and Results sections). 
jointly exhaustive in terms of accounting for each and every movement that was observed for the species. The development of ethograms capable of these tasks was accomplished by first using several carefully formulated criteria in order to develop preliminary ethograms, then by refining the ethograms and criteria used to identify specific movement patterns, and finally by conducting interrater reliability tests of the ethograms. These methods are discussed next.

Criteria used to generate the preliminary ethograms. Based on observations made during the videotaping and on subsequent observations of approximately $30 \%$ of the videotapes chosen at random, a preliminary ethogram for each species was created. Movement patterns representing different categories were discriminated from each other by using the following criteria. Two movement patterns represented different movement pattern categories if: (1) they involved movements of different articulations and muscles, e.g., "locomoting-normative (slow rhythmical) gait" versus "chewing grass," (2) they involved movements of the same articulations but the pattern of movement around the articulations was spatiotemporally distinct, e.g., "locomoting-normative (slow rhythmical) gait" could be discriminated from "locomoting-trotting or running speed" based on differences in limb trajectories and limb movement speeds, and (3) they involved movements around the same articulations and the pattern of movement around the articulations was spatiotemporally similar, but the movement patterns were associated with contextually or functionally distinct cues, e.g., "chewing grass" events were always preceded by an animal's acquiring a bite of grass, whereas "ruminating" events were always preceded by a visually detectable regurgitation or peristaltic movement that could be seen along the ventral surface of an animal's neck.

Based on these 3 criteria, movement pattern categories were created that could be used reliably to identify movement patterns made by each animal on a second-by-second basis for the full 6-15 h videotaped session. These movement pattern categories represented the preliminary ethograms that were then refined as discussed next.

Ethogram refinement. Two investigators initially naive to the study were asked to generate their own lists of movement pattern categories based on the following criteria: (1) a discrete movement pattern had to have a visually discernible onset and offset, (2) occurrences of movement patterns representing the same movement pattern category had to involve the same general set of articulations and muscles during each occurrence, (3) occurrences of movement patterns representing the same movement pattern category had to appear similar, in spatiotemporal terms, during each occurrence, (4) occurrences of movement patterns representing the same movement pattern category had to have observably similar functions, as deduced from contextual information associated in space or time with the movement, (5) a specific movement pattern category could not be ambiguous, i.e., any movement in which the animal engaged had to be definable by one and only one movement pattern 
category; recurrent ambiguities between two or more categories necessitated combining them into a single category, (6) the ethogram or list of movement pattern categories had to be jointly exhaustive, meaning that every movement in which an observed animal engaged had to be definable by a category on the list.

The naive investigators then viewed videotapes of all six species involved in the study. While watching the videotapes, the naive investigators generated their own ethograms for each species. During the time that both naive investigators were creating these ethograms, they did not have access to the preliminary ethograms nor to each other's findings.

After the naive investigators' ethograms were created, they were compared with the preliminary ethograms. The lists created by the naive investigators as well as the preliminary ethograms were then compiled into a single set of ethograms agreed upon by all investigators involved in the ethogram creation procedure, and this final ethogram set appears as Table 2.

It is important to state that several movement pattern categories could be used simultaneously to describe what an animal was doing. For instance, if an okapi moved its tail, licked its lips and moved its head simultaneously, then movement patterns representing three different categories, viz., "adjusting or moving tail," "licking lips with tongue," and "adjusting or moving head and/or neck," were said to be occurring simultaneously. Only if movements involving sevcral joints and limbs always co-occurred, e.g., "locomotion," was a single category used to define this movement. This procedure provided an objective means of defining, in an abbreviated or integrated form, complex movement patterns involving multiple joints and appendages.

Interrater and intrarater reliability tests. Interrater and intrarater reliability tests were performed to estimate the reliability of the methods employed in the current investigation. The interrater reliability test involved performing sporadic spot checks ( $n-300$ total times per naive investigator) by one of us (GEG) during continuous focal record creation. During a spot check, the videotape that was being viewed by a naive investigator was put on pause by GEG, who was blind to what was currently being viewed on the tape. This served to isolate reasonably random segments of videotapes.

Because the animals spent a majority of the time resting, videotape segments selected in this way usually showed an animal at rest, an activity that was always easy to define by different investigators. Therefore, in order to reflect a broader range of an animal's movement patterns during these spot checks, the videotape was restarted, and the next movement engaged in by the animal was used in the spot check.

During a spot check, both GEG and the naive investigator wrote down the movement pattern category or categories that best described what the animal was doing during the selected videotape segment. Both investigators were blind to each other's choices. Next, both the investigators' choices were compared, and a match occurred when both had written down the same 
category or categories. The number of matches, expressed as a percentage of the total number of spot checks became the interrater reliability test scores. For one naive investigator, the interrater reliability test score was $96 \%$ and for the other naive investigator $100 \%$.

Intrarater reliability tests of movement pattern event durations were done at the same time as the interrater reliability spot checks. These tests indicated that event durations were accurate to within $\pm 0.1 \mathrm{~s}$ over $95 \%$ of the time. These results are similar to those previously reported in other studies (Kien et al. 1991).

\section{Continuous Focal Records}

Continuous focal record format. Videotaped behavior data were converted to continuous focal records (Martin and Bateson 1986) and stored as computer files (IBM PS/2 Model 70) using software developed by one of us (GEG). For purposes of this study, each movement pattern category was conceived of as a channel running continuously in time (Figure $1 A$ ), and during a videotaping session, each animal engaged in movements that could be classified by one of many different movement pattern categories. Figure $1 A$ shows five possible movement pattern categories or channels and how movement pattern events were recorded in each channel. Movement pattern events are described below and in Figure $1 B$.

Movement pattern events. Whenever an animal movement occurred, an investigator pressed a keyboard key corresponding to the appropriate movement pattern category that best described the movement. The movement pattern category channel was then turned on for as long as the animal continued the same movement (Figure $1 A, B$ ). Thus movement onsets and offsets were precisely recorded. Movement pattern event durations were defined as the time from onset to offset of an event in a specific movement pattern category channel (Figure $1 B$ ). All of the results of the current study are based on such event duration measurements. Based on software precision, event durations as short as $0.01 \mathrm{~s}$ were recordable.

The software also allowed the user to enter multiple movement pattern categories simultaneously or even asynchronously for times when an animal engaged in more than one movement pattern concurrently. An investigator could also indicate when the animal was partly or totally hidden, and whether movement responses corresponded to some overt external environmental stimulus such as a loud noise.

Sampling methods. Random sample periods of the videotapes were selected by first dividing a videotaping session, i.e., 6-15 h of continuously recorded videotape, into $2 \mathrm{~h}$ periods (Figure 2). Then, random times from each $2-\mathrm{h}$ period were selected by using software developed in the laboratory. These randomly selected times defined the time at which data record collection 


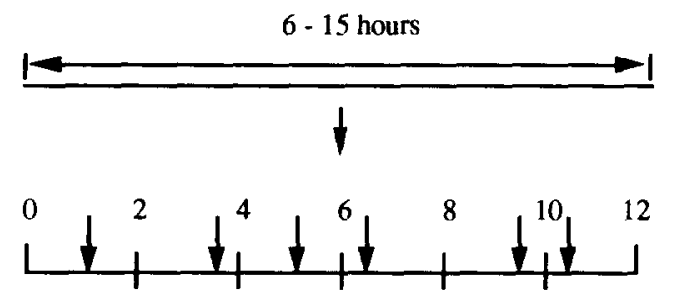

Videotape session

duration

Videotape session divided into equal $2 \mathrm{hr}$ periods, and random times selected from each period (arrows)

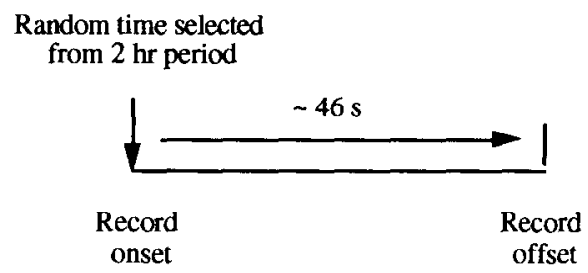

FIGURE 2. Method of data sampling and continuous focal record creation (see text).

started (Figure 2). The program was specifically designed to avoid creating sample periods that were spaced evenly in time so that possible ultradian rhythm effects were minimized. A given videotape was turned on at the time designated by the software, and a continuous focal record was made while the tape ran at $0.1 \times$ normal viewing speed. As the videotape ran, the computer clock kept track of time with respect to the videotape speed. In other words, when the videotape ran at $0.1 \mathrm{x}$ speed, then the computer clock also ran at $0.1 \mathrm{x}$ speed. Record creation then continued for $7 \mathrm{~min}$ or until at least three total events had been recorded, whichever was longer. This technique assured that events with relatively long durations would be recorded.

Records always began with the onset of a movement pattern event and ended with another movement pattern event's offset. Hence, no movement pattern event durations were underestimated by record creating procedures. The continuous focal records in this way averaged $46 \mathrm{~s}$ in actual elapsed time on the videotape (Figure 2), and for each record the number of different movement pattern event entries ranged from 3-103.

Sequencing of continuous focal record creation. The order in which the continuous focal records were created was randomized by computer program, which was designed to minimize clumping of the same individual animal, or of the same species. In this way, any effects associated with changes in an investigator's data collection abilities would be randomized across individuals within a species and across species.

\section{Statistics}

Event durations (Figure $1 \mathrm{~B}$ ) were used in summary statistics to evaluate the variable's range of values among the six species. Event durations that were 


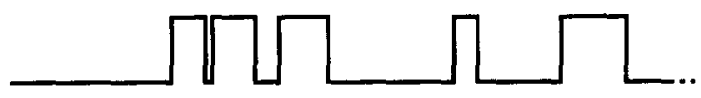

Adjusting or moving head and/or neck

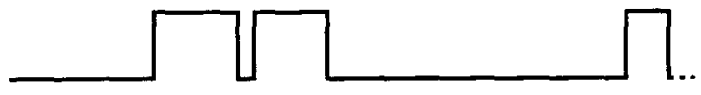

Licking lips with tongue

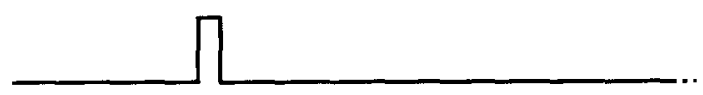

Standing still-eyes open

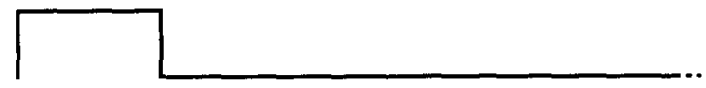

Locomoting-normative (slow rhythmical) gait
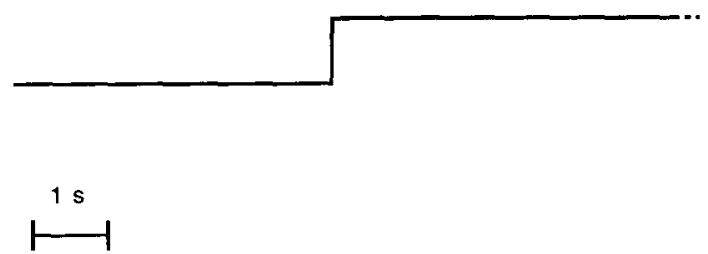

FIGURE 3. A segment from a continuous focal record made of an okapi.

collected by one of the two naive investigators were subjected to a nested design, one-way classification, mixed effects model, unequal sample size analysis of variance, using Satterthwaite's approximate F-test for testing purposes (Dunn and Clark 1987). This statistical analysis was designed to test for the equality of event duration means among the six different species and among the different movement pattern categories within species. The software used to conduct this analysis was created and thoroughly tested in the laboratory. Prior to this analysis of variance, data were log transformed so that sample sizes were normally distributed (Wilk's test for normality). Normality tests were performed using BMDP 2D (Engleman 1990). Events that were associated with loud noises or with the animal's being partly or totally hidden from view (see "Movement pattern events," above) were excluded from all statistics described above.

\section{RESULTS}

\section{A Representative Continuous Focal Record}

Figure 3 depicts a segment from a continuous focal record that was created by one of the naive investigators (see Methods). The figure shows, in time series format, $8.17 \mathrm{~s}$ of data from a record that was $29.64 \mathrm{~s}$ in total duration. Five movement pattern category channels are shown; these are "Adjusting or moving ear(s)," "Adjusting or moving head and/or neck," "Licking lips with 
tongue," "Standing still-eyes open," and "Locomoting-normative (slow rhythmical) gait." Note the time scale at the bottom of the figure.

Figure 3 demonstrates the complexity that often arose in the data during periods when an animal was active. For instance, note that after the initial "Standing still-eyes open" event, an "Adjusting or moving head and/or neck" event occurred. During the course of this "Adjusting or moving head and/or neck" event, two "Adjusting or moving ear(s)" events, and one "Licking lips with tongue" event also occurred. Note that these latter three events' onsets and offsets did not coincide with the "Adjusting or moving head and/or neck" event's onset or offset (Figure 3). In other words, events representing different movement pattern categories often occurred relatively independently of each other. Data were often complex in that events in many different channels overlapped in such asynchronous ways. However, the software used to digitize the movement events made it possible to collect accurate event onsets and offsets when viewing the videotapes at $0.1 \times$ normal speed (see Methods).

Despite the complexity that existed across all active movement pattern category channels, the activity within a single channel was easily followed. For instance, note that in Figure 3, there are five "Adjusting or moving ear(s)" events, three "Adjusting or moving head and/or neck" events, one "Licking lips with tongue" event, one "Standing still-eyes open" event, and finally one "Locomoting-normative (slow rhythmical) gait" event, which continues after the end of the depicted segment. The events in any one of these channels are easy to track, and each event has a specific duration (Figure 3). Such event durations represented the variable analyzed in this study (see below).

\section{Event Duration Results}

Figure 4 is a histogram showing the distribution of movement pattern event durations for the six giraffes. The $x$-axis is time in seconds on a logarithmic scale. Note that as a result of the logarithmic scale, bins further from the origin represent increasingly larger time spans. For instance, the bin nearest the origin ("0.00-0.09") represents a range of $0.09 \mathrm{~s}$; however, the bin farthest from the origin ("63.10-99.99") represents a range of 36.89 s (Figure 4).

The $\mathrm{z}$-axis in Figure 4 represents the individual movement pattern categories observed in the six giraffes, and each category is treated separately on this axis. In other words, the data shown in one movement pattern category bin, e.g., "Tongue stereotypy," found in the foreground on the z-axis of Figure 4, were treated independently of other movement pattern categories, e.g., "Hiccuping/twitching," "Pacing-slow," etc.

The y-axis or vertical axis in Figure 4 is labeled "\% total events within a category." The $y$-axis label emphasizes the fact that percentages are based on the total number of events representing each movement pattern category separately. For instance, in the category, "Hiccuping/twitching" (Figure 4), all events had a duration of 1.00-1.57 s. Thus, $100 \%$ of "Hiccuping/twitching" events were $1.00-1.57 \mathrm{~s}$ in duration as the bar indicates. 


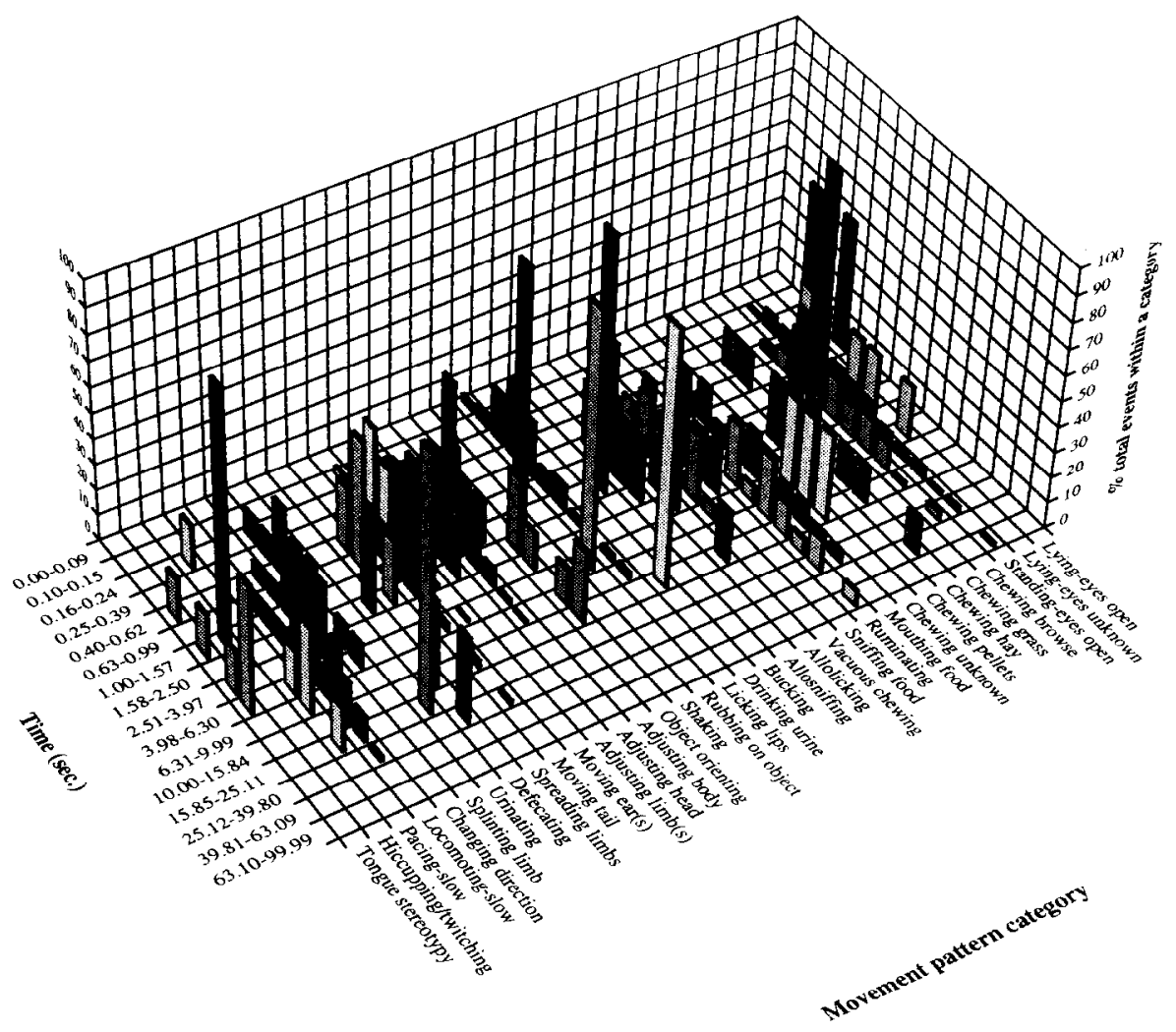

FIGURE 4. Percentage distributions of event durations for the giraffes (see text). These giraffe movement pattern category results are based on a total of 1335 events for all categories combined. Minimum events per category $=1$; maximum events per category $=$ 479 ; mean events per category $=39.26$ for the giraffes.

To construct Figure 4, event durations for all six giraffes were pooled by movement pattern category. Next, the total number of events representing each movement category was calculated. Finally, the number of events with durations occurring within each $x$-axis time bin ("Time (sec.)," Figure 4) was determined and expressed as a percentage of the total number of events representing the same movement category. Thus, Figure 4 is a three-dimensional histogram of event durations, where the third dimension is used to show results for individual movement categories. Although pooling the data across all categories would have resulted in a more standard two-dimensional histogram showing the distribution of all giraffe event durations combined, it was felt that more detail regarding individual categories could be appreciated using the format shown in Figure 4.

Although the results in this figure represent giraffe event duration data, the results are representative of event duration data for the other five species. Event sample sizes per species ranged from 544 events for pandas to 1381 events for kangaroos. Mean sample sizes per movement pattern category ranged from 20.92 events for Red pandas to 46.03 events per category for 
kangaroos; sample sizes ranged from $1-479$ events per movement pattern category.

The entire set of event durations was not tested statistically due to the complexity of the data collection procedures, i.e., unequal sample sizes between movement pattern categories and hetween species, plus the effects due to different investigators (Gerstner 1992). However, data collected by one investigator ( $n=3707$ events) were subjected to a nested design, one-way classification, mixed effects model, unequal sample size analysis of variance, using Satterthwaite's approximate F-test for testing purposes. The results of this test found no significant differences among the six species' mean event durations $(\mathrm{F}[5,1515.433]=0.785, p>.05)$. However there were significant differences within species among the mean event durations of the different movement pattern categories $(F[111,3560]=17.814, p<.0005)$.

There were three important findings contained in the individual species plots, of which Figure 4 is a representative. Firstly, note that the maximum event durations for most movement pattern categories was $<25 \mathrm{~s}$. Mean event durations for each species were $1.6 \mathrm{~s}-4.8 \mathrm{~s}$ (see below). In other words, all six species were characterized by relatively short duration events.

Secondly, note that the longest event durations generally were those belonging to categories associated with inactive categories or vegetative states, e.g., the "Standing" and "Ruminating" categories, Figure 4. These categories frequently occurred during pcriods of "sleep" or long episodes of rest in the animals. In other words, sleeping or resting animals were characterized by longer event durations.

Finally, note that the shape of many of the curves is fairly symmetrical on the logarithmic plot of each movement pattern category (Figure 4), and that few of the curves appear skewed to the left. Since the continuous focal records, from which Figure 4 was made, averaged about $46 \mathrm{~s}$, there was the possibility that longer events could have been missed as a result of the sampling methods. If event durations had actually averaged $\geq 40 \mathrm{~s}$, then most of the curves should have shown left skewing, with sharp cutoffs after the bin labeled "39.8163.09." The fact that this did not occur provides evidence that the employed sampling methods represented the actual event duration distributions fairly well.

Figure 5 is a summary of the data shown in Figure 4 , as well as a summary of similar data from the other five species. The six species are listed vertically along the left side of this figure. Time is represented along the horizontal axis; note the logarithmic plot of time (Figure 5). In order to create this figure, first the mean event duration for each of the movement pattern categories for a given species was calculated. Then the mean of all movement pattern category means for each individual species was calculated and plotted (filled circles in Figure 5). In addition, the shortest and longest events from all categories pooled within a species were determined and plotted (asterisks in Figure 5). Thus, this plot shows the entire range of event durations from the six different species. Furthermore, the plot summarizes event duration data from 


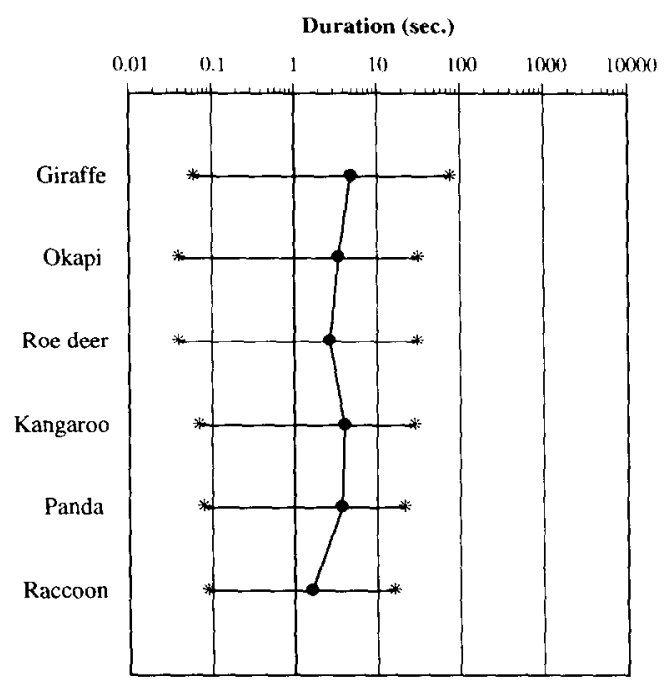

FIGURE 5. Minimum event duration, maximum event duration, and mean of all category event duration means for each of the six species. Filled dots = means of all category event duration means for each species. Asterisks to the left and right of each dot represent, respectively, the minimum and maximum event durations for all categories combined within each species.

26-37 different movement pattern categories per species (see footnote \# 4 under Table 2). Figure 5 is based on a total of 6039 events and uses the data collected by both naive investigators.

These results (Figure 5) show that mean event durations and ranges for the six species were similar, corroborating the results of the analysis of variance presented above. The means of all category event duration means (Figure 5) \pm 1 standard error of the mean for each species were:

Giraffes $=4.82 \mathrm{~s} \pm 4.99 \mathrm{~s}$

Okapis $=3.42 \mathrm{~s} \pm 4.06 \mathrm{~s}$;

Rue deer $=2.70 \mathrm{~s} \pm 3.00 \mathrm{~s}$;

Kangaroos $=4.04 \mathrm{~s} \pm 6.04 \mathrm{~s}$;

Pandas $=3.76 \mathrm{~s} \pm 5.13 \mathrm{~s}$;

Raccoons $=1.62 \mathrm{~s} \pm 1.65 \mathrm{~s}$.

\section{DISCUSSION}

\section{Summary of Results}

A major goal of this project was to account for every gross movement that free-roaming animals made over the course of 6-15 h time periods in relatively rich environmental settings. By accounting for every movement and then evaluating the range of event durations associated with every movement, it was believed that an appreciation for and some understanding of the physiological constraints on movement event durations could be achieved. 
With this in mind, the results showed that mean event durations \pm 1 standard error of the mean for each species ranged from $1.62 \mathrm{~s} \pm 1.65 \mathrm{~s}$ for raccoons to $4.82 \mathrm{~s} \pm 4.99 \mathrm{~s}$ for giraffes. Thus, mean event durations in all species were on the order of a few seconds and were not significantly different across the six species $(\mathrm{F}[5,1515.433]=0.785, p>.05$ ).

These findings are all the more surprising considering the range of species included in the study and the range of conditions present during the study. For instance, in terms of size, the giraffe is one of the largest terrestrial mammals (Eisenberg 1981, pp. 464-471), and the individuals used in the current study weighed about $1100 \mathrm{~kg}$ and averaged about $4.5-5 \mathrm{~m}$ in height. These dimensions stand in stark contrast to the Red pandas, which averaged about $3.5 \mathrm{~kg}$ and were about $0.5 \mathrm{~m}$ from snout to tail tip. Indeed, the giraffe's head (15 kg, Powell 1984) is more than 4 times heavier than the total body weight of the panda. Furthermore, the six species studied represented a considerable range of niches, phylogenetic histories, and different zoogeographic regions (see Eisenberg 1981). Thus, it is indeed remarkable that, despite the clear differences among the species, the mean event durations of the six species were not statistically significantly different.

In conjunction with this, the animals were studied under a broad range of conditions. For instance, at the Phoenix Zoo, winter morning hour temperatures were often as low as $-2^{\circ} \mathrm{C}$, whereas summer mid-day temperatures at all four zoological institutions were occasionally as high as $46^{\circ} \mathrm{C}$. Furthermore, rainstorms, windstorms, and extreme arid conditions sometimes occurred immediately before or during videotaping sessions. Light conditions also varied significantly during videotaping sessions. In the early morning and late evening, light levels were in marked contrast to light levels available during the mid-day hours.

Enclosure conditions varied significantly as well among the six species (Table 1). For instance, the Rothschild's giraffes' 128 acre natural habitat, which contained a broad variety of flora and fauna indigenous to east Africa, was very different from the Masai giraffes' $350 \mathrm{~m}^{2}$, relatively barren enclosure. Finally, it is important to recognize that parental care of and group interaction with new babies was recorded for several species (see Methods). Despite all of these potential influences on movement pattern event durations, the range of observed event durations was relatively narrow (Figures 4,5 ).

Whether the ranges shown in Figure 5 approximate the minimum and maximum event durations that each species is capable of is not known. Further investigations in this area will be required in order to evaluate the flexibility inherent in event durations between different movement patterns and between different species.

\section{Comparison of the Current Study's and Previous Studies' Results}

The fact that the mean event durations for the six mammalian species studied was $1.62 \mathrm{~s}-4.82 \mathrm{~s}$ is compelling in light of previous work in human motor 
skills. The current study's definition of an event (see Methods and Figure $1 \mathrm{~B}$ ) is the same as Schleidt's (1988) definition of a behavior episode. In Schleidt's (1988) study, "the duration of the whole repetitive behavioral act" was measured. Examination of Schleidt's methods (1988) indicates that this measure was equivalent to event durations in the current study.

Furthermore, Schleidt's (1988) behavioral acts included caressing movements with the hands, crawling movements, hopping movements, etc. These "behavioral act" definitions were similar to many of the current study's movement pattern categories, e.g., grooming, locomoting (Table 2). Hence, not only is Schleidt's definition of a behavior episode the same as our definition of a movement category event, but many of her definitions of acts were similar to our definitions of movement categories.

With this in mind, Schleidt (1988) found that behavioral acts in humans had durations of about $3 \mathrm{~s}$, a finding very much in agreement with the current study's results of event durations. Furthermore, her data (Figure 1 in Schleidt 1988) showed that the durations of human behavioral acts were $<17 \mathrm{~s}$. By the same token, the current study found that most event durations were $<25 \mathrm{~s}$ for the six mammalian species (Figure 4). It must be emphasized that the two naive investigators that were recording and entering data in our study (see Methods) were blind to Schleidt's results. They were also blind to all previous work reporting a $3 \mathrm{~s}$ time constant in human perception (Pöppel 1972, 1973, 1978, 1988; Woodrow 1951), human speech (Turner and Pöppel 1983), and chimpanzee motor tasks (Kien et al. 1991). With this in mind, it is extremely compelling that the current study and previous studies arrived at similar results for different species.

Because most of the previous psychophysical studies (Schleidt et al. 1987; Schleidt 1988; Woodrow 1951; Pöppel 1978) have focused on the role of short duration time constants in human skills, their conclusions have necessarily emphasized the human aspect and function of the $3 \mathrm{~s}$ time constant. In Schleidt's (1988) words: "There are two possible functions of this universal [3 s] time constant. Firstly, the building up of a subjective present and a feeling of 'now'-ness might have the 3-s time constant as a prerequisite (Pöppel 1988). Secondly, communication and synchronization between people might be facilitated by a mechanism which creates a common time beyond the content of a behavior pattern."

More recently, it has been shown that chimpanzee hand movements are also segmented into action units with median durations of approximately $2 \mathrm{~s}$ (Kien et al. 1991). Kien et al. (1991) concluded that short duration temporal segmentation is not unique to human language ability. Rather, temporal segmentation is a preexisting feature of the pongid brain upon which language, human perception, and motor skills have been elaborated.

Results of the current study suggest that the $3 \mathrm{~s}$ time constant and the physiological mechanisms subserving this time constant are even more ancestral, extending into non-primate lineages as well. That is, the $3 \mathrm{~s}$ time constant could be the signature of a physiological process that is found in many 
different mammalian species and orders. Such a finding would be consistent with evolutionary and ethological theory (e.g., cf. Lorenz 1981, pp. 72-103) as discussed below.

\section{Homology and the $3 \mathrm{~s}$ Time Constant}

Evolutionary theory suggests that many complex morphological features are conserved in evolutionary time scales (Lorenz 1981, pp. 72-103). Bones like the radius, ulna, and humerus are identifiable in many mammalian species (e.g., Lorenz 1981, fig. 9, p. 94). Such bones have evolved to fulfill different functions in different species; however, the preservation of complex features on the bones (e.g., tuberosities, condyles, trochanters, etc.) serves to identify the same bones in different species. Bones with a common ancestry in different species that have evolved to serve different functions in different species are said to be homologous.

Furthermore, classical ethology suggests that the concept of homology can be extended to include behavior patterns (Lorenz 1981, pp. 107-110). That is, certain spatial or temporal characteristics of behavior patterns can be used to identify homologous movement patterns in different species (Lorenz 1981, p. 107). With this in mind, the $3 \mathrm{~s}$ time constant associated with movement patterns in humans (Schleidt et al. 1987; Schleidt 1988), chimpanzees (Kien et al. 1991), giraffes, okapis, Roe deer, Red Pandas, Eastern gray kangaroos, and raccoons could be the manifestation of an underlying physiological process that is homologous among these different mammalian species.

If the $3 \mathrm{~s}$ time constant is the manifestation of a homologous neurophysiological system operating in different mammalian species, then this neurophysiological system has obviously been adapted to new functions in different species, just as mammalian bones can be adapted to new and different functions. For instance just as the radius, ulna, and humerus have been adapted for flight in bats, locomotion in quadrupeds, brachiation in some primates, and swimming in whales, the $3 \mathrm{~s}$ time constant has been adapted to the functions of the "feeling of "now'-ness" and "communication and synchronization between people" (Schleidt et al. 1987; Schleidt 1988), and to a variety of evolutionarily elaborated motor programs in different mammalian species (Table 2, Kien et al. 1991). Thus, the $3 \mathrm{~s}$ time constant could be considered a universal and ubiquitous organizing process of mammalian movement patterns. This possibility suggests that further comparative ethological work in this area will be important and compelling.

\section{Toward a Neuroethology of the $3 \mathrm{~s}$ Time Constant}

The neurophysiological nature of the $3 \mathrm{~s}$ time constant is currently unknown. However, in light of the current study's findings, it is clear that animal models may now be developed so that the anatomic and physiologic nature of the $3 \mathrm{~s}$ 
time constant can be investigated. This is one promising direction for future studies.

We hypothesize that the $3 \mathrm{~s}$ time constant likely involves many central neural mechanisms and that most if not all of these mechanisms will be located anatomically at levels higher than the brain stem. This is based on the following findings and assumptions. First, the subjective present (Pöppel 1978; Woodrow 1951) with which the $3 \mathrm{~s}$ time constant is associated is a very complex perceptual skill. It is believed that such complex perceptual skills involve higher central neural systems (Ruch 1951a). Second, there is strong evidence that human motor bchaviors likc those observed by Schleidt et al. (1987) and mammalian movement patterns like those observed in the current study involve neural centers at levels higher than the brain stem (for reviews, see Ruch 1951b; Bock et al. 1987). Hence, evidence from several independent sources suggests that higher neural centers are involved in movement pattern event durations.

Furthermore, recent work by Lin et al. (1991) showed that cooling of the somatosensory cortex lengthened masticatory event durations. Based on this work, we suggest that the cortex plays an important and possibly fundamental role in movement pattern event durations. However, it is likely that other central neural systems will also be involved (Gerstner 1985). Future work will be required to determine the precise nature of the mechanisms associated with event durations. Thereafter, comparative work can be done to determine whether, in fact, similar neurophysiological mechanisms subserve event durations of different movement patterns within a species and of different movement patterns in different species.

In summary, the current study provided strong evidence for a $3 \mathrm{~s} \mathrm{time}$ constant associated with movement pattern event durations in 6 diverse mammalian species. This time constant was documented previously in human perceptual (Pöppel 1973, 1978) and motor skills (Schleidt et al. 1987; Schleidt 1988), and in motor skills of chimpanzees (Kien et al. 1991). The results of the current study suggest that the $3 \mathrm{~s}$ time constant is the signature of a neurophysiological system that has been highly conserved at least at the mammalian class level. Future work will be required in order to determine whether this $3 \mathrm{~s}$ time constant is indeed subserved by the same neurophysiological systems in different species, and if so at what taxonomic level-i.e., mammal, vertebrate, chordate-the time constant is conserved.

We thank K. Berridge, T. Lee, M. Schleidt, and an anonymous reviewer for important comments and critical reviews of this article. We would like to thank Jeaniene Talley and Derrick Kubota for assisting with data acquisition and analysis. We thank the staffs of the Greater Los Angeles Zoo, Phoenix Zoo, San Diego Zoo, and San Diego Wild Animal Park for access to and information regarding the animals. This study was supported by NIH grant DEI00205. 


\section{REFERENCES}

Bock, G., O'Connor, M., and Marsh. J. (Eds.). Motor Areas of the Cerebral Cortex, Chichester: John Wiley and Sons, 1987.

Boever, W. J., and Leathers, C. Pulmonary and encephalic infection secondary to lumpy jaw in kangaroos. Journal of Zoo Animal Medicine 4: 13-16, 1973.

Dunn, O. J., and Clark, V. A. Applied Statistics: Analysis of Variance and Regression, New York: John Wiley and Sons, 1987.

Eisenberg, J. F. The Mammalian Radiations, Chicago: The University of Chicago Press, 1981.

Engleman, L. Detailed data description including frequencies. In BMDP Statistical Software Manual, Vol. I, W. J. Dixon (Ed.). Los Angeles: University of California Press, 1990, pp. 135-144.

Feldhütter, I., Schleidt, M., and Eibl-Eibesfeldt, I. Moving in the beat of seconds; analysis of the time structure of human action. Ethology and Sociobiology 11: 511-520, 1990.

Gerstner, G. E. An ethological approach to the study of neuroleptic-induced oral dyskinesias in guinea pigs: special emphasis on tardive dyskinesia. Master's thesis, University of California at Los Angeles, 1985.

Gerstner, G. E. Quantifiable temporal and spatial behavior patterns in six mammalian species: insights into ultimate and proximate causality. Ph.D. dissertation, University of California at Los Angeles, 1992.

Gwinner, E. Circannual Rhythms, Endogenous Annual Clocks in the Organization of Seasonal Processes, Berlin: Springer-Verlag, 1986.

Kavanau, J. L., and Peters, C. R. Primate locomotor pattern repetitions, program clocks, and orientation to light. Primates 15: 209-217, 1974.

Kavanau, J. L., and Rischer, C. E. Program clocks in small mammals. Science 161: 1256-1259. 1968.

Kien, J., Schleidt, M., and Schöttner, B. Temporal scgmentation in hand movements of chimpanzees (Pan troglodytes) and comparisons with humans. Ethology 89: 297-304, 1991.

Lin, L. D., Murray, G. M., and Sessle, B. J. Effects of cooling face somatosensory cortex on primate mastication. Journal of Dental Research 70: 465, 1991.

Lorenz, K. The Foundations of Ethology, New York: Simon and Schuster, 1981.

Martin, P., and Bateson, P. Measuring Behavior, New York: Cambridge University Press, 1986.

Pöppel, F. Oscillations as possihle hasis for time perception. In The Study of Time, J. T. Fraser, F. C. Huber, and G. H. Müller (Eds.). Berlin: Springer-Verlag, 1972, pp. 219-241.

Pöppel. E. Influence of pause duration on the reproduction of a 2-second interval. Bulletin of the Psychonomics Society 2: 291-292, 1973.

Pöppel, E. Time perception. In Handbook of Sensory Physiology, Vol. VIII, R. Held, H. W. Leibowitz, and H. L. Teuber (Eds.). Heidelberg: Springer-Verlag, 1978, pp. 713-729.

Pöppel, E. Mindworks: Time and Conscious Experience, Boston: Harcourt Brace Johanovich. 1988.

Powell, R. A. Giraffe and okapi. In The Encylopedia of Mammals. D. MacDonald (Ed.). New York: Facts on File Publications, 1984, pp. 534-541.

Ruch, T. C. Sensory mechanisms. In Handbook of Experimental Psychology, S. S. Stevens (Ed.). New York: John Wiley and Sons, 1951a, pp. 121-153.

Ruch, T. C. Motor systems. In Handbook of Experimental Psychology, S. S. Stevens (Ed.). New York: John Wiley and Sons, 195lb, pp. 154-208.

Schleidt, M., and Feldhütter, I. Universal time constant in human short-term behavior. Naturwissenschaften 76: 127-128, 1989.

Schleidt, M. A universal time constant operating in human short-term behaviour repetitions. Ethology 77: 67-75, 1988.

Schleidt, M., Pöppel, E., and Eibl-Eibesfeldt, I. A universal constant in temporal segmentation of human short-term behaviour. Naturwissenschafien 74: 289-290, 1987.

Turner, F.. and Pöppel, E. The neural lyre: poetic meter, the brain and time. Poetry 277-309, 1983.

Wilson, E. O. Sociobiology, Cambridge: The Belknap Press of Harvard University Press, 1980.

Woodrow, H. TIme perception. In Handbook of Experimental Psychology, S. S. Stevens (Ed.). New York: John Wiley and Sons, 1951, pp. 1224-1236. 